\title{
Merkel Cell Carcinoma: A Case-Report of Rare Presentation of Merkel Cell Carcinoma, Non-Indolent Proliferative Type, Polyomavirus-Negative: Hypothesis of Molecular- Pathogenesis
}

\author{
West Michele ${ }^{1 \star}$, Findakly Dawood ${ }^{2}$ and Amar Surabhi ${ }^{3}$ \\ ${ }^{1}$ Department of Internal Medicine, Norwalk Hospital, Yale, USA \\ ${ }^{2}$ Creighton University/ Maricopa Integrated Health System, \\ Internal Medicine Department
}

${ }^{3}$ Creighton University/Maricopa Hematology Oncology Department
${ }^{*}$ Corresponding author

West Michele, Department of Internal Medicine, 32 President Street, Hempstead N.Y. 11550, Number: (405) 614-8374, Fax: (516) 385-1746, Norwalk Hospital, Yale, USA

Submitted: 10 Mar 2020; Accepted: 17 Mar 2020; Published: 26 Mar 2020

\begin{abstract}
Introduction: Merkel cell carcinoma (MCC) is a rare cutaneous indolent neuroendocrine cancer, arising from the Merkel cells of the stratum basalis, of the epidermis. This type of tumor commonly arises in sun-exposed areas, such as head, neck, and lower extremities. Here, we describe a rare presentation of non-indolent proliferative type Merkel cell carcinoma.

Case Report: This is a case-report of a 70-year-old Caucasian man with no pertinent past medical history, presenting as a large painless violaceous necrotic irregular bordered rapidly growing lesion, reaching to $8 \times 10 \times 15 \mathrm{~cm} 3$, within one-year period, on the lower back region with surface ulceration. Pelvic MRI showed a multilobulated enhancing soft tissue mass measuring $8.7 \times 10.4 \times 15.1 \mathrm{~cm} 3$ at the left gluteal region with exophytic extension to the left paraspinal muscles. The patient was admitted, tangential surgical excision and debridement of left flank wound was performed with partial primary closure. Pathology showed features of Merkel cell, show diffuse dot-like positivity with CK20 and are negative for CK7, there is diffuse positivity with NSE, synaptophysin and CD56 with strong diffuse Ki-67 positivity noted in $>65 \%$ of tumor cells. CD99 shows diffuse small faint dot-like paranuclear positivity.

Discussion: Merkel cell carcinoma (MCC) is a rare, aggressive tumor that generally arises in sun-exposed regions. After an initial course of slow growth, starting as a painless violaceous non-pruritic domed-shaped lesion, the tumor becomes more aggressive, rapidly growing, with metastasis with local lymph nodes and regional tissue invasion. Sixty percent of tumors can rapidly grow within a three-month period after initial diagnosis. MCC is also clonally associated with is polyomavirus. MCC Contributing molecular pathogenesis is imperative to determining the causation of rare non-indolent MCC tumors, and its association with prognosis and treatment. In polyomavirus negative patients, consideration for molecular pathogenesis as etiology is imperative.
\end{abstract}

Keywords: Merkel Cell Carcinoma, Merkel Cell, Neuroendocrine, Neuroendocrine Tumor, Polyomavirus, Skin Cancer, Molecular Pathogenesis

\section{Introduction}

Merkel cell carcinoma (MCC) is a rare, aggressive, cutaneous and indolent neuroendocrine cancer, arising from the merkel cells of the stratum basale. These are cells of neural crest origin found in the dermis, first describe by Friedrich Merkel in 1875, and function as somatosensory fibers in low frequency vibrations [1]. This tumor usually arises in sun-exposed areas, such as head, neck, and lower extremities. It typically starts as a painless, violaceous, non-pruritic, intradermal nodule, although can also start as an ulcerated, cystic or acneiform lesion. It generally increases rapidly in size over a period of weeks or months with a tendency for local recurrence (20-75\%), regional nodal metastasis (31-80\%) and distant metastasis (26-75\%) $[1,2]$. Tumors as small as $2 \mathrm{~cm}$ have been reported to metastasize with a mortality of $33 \%$. Equally affects both sexes, but more common in whites with $<5 \%$ cases, less than $65 \%$ affecting white males, living commonly in northern Europe and United States, at a 2:1 ratio male to female, with adjustment made for age, there is a direct correlation with UVB radiation exposure, as well as, time of exposure $[1,3]$. Survival rates depend on metastasis and distance of disease, with local occurrence with approximately $50 \%$ survival, to less than $14 \%$ survival with distant metastasis [4].

MCC often occurs in immuno-compromised patients like those with HIV/Hepatitis infections, organ transplant recipients or patients with chronic lymphocytic leukemia. MCC is also clonally associated with polyomavirus, while a small number of cases are polyomavirus negative, associated with other molecular pathogenesis [3]. Polyomavirus integrates in the cell DNA, causing tumorigenesis through modification in cell antigen expression, inhibiting lymphocytic recognition of tumor cells. MCC diagnosis can often 
be delayed due to benign appearance of initial lesion/misdiagnosis. Histologic exam shows a trabeculated and solid patterns of malignant cells infiltrating the dermis. These are uniform, small blue cells with finely granular nuclear chromatin and numerous mitotic figures [5]. The differential diagnosis is anaplastic carcinoma and small cell cancer of the lung (SCLC). Stains for neuron-specific enolase, chromogranin are positive and Electron microscopy shows neurosecretory granules. Treatment usually involves wide surgical excision (with a $2 \mathrm{~cm}$ margin) with sentinel node (SLN) mapping. Those with positive SLN undergo radical lymph node dissection with subsequent radiation. Chemotherapy involves drugs commonly used in SCLC like platinum and etoposide [2,4]. Also, immune check point inhibitors have been found effective in metastatic cases. Through combination of molecular pathogenesis and morphological pathology, a new basis of treatment and management of MCC can be formed with an established polyomavirus status [6].

\section{Case Report}

A 70-year-old Caucasian male, lifer/prisoner, with past medical history of hyperlipidemia and diabetes mellitus, non-compliant, with HgbA1C of $8.6 \%$, presented with a large painless violaceous necrotic irregular bordered rapidly growing lesion, reaching to $8 \times 10 \times 15 \mathrm{~cm}^{3}$, within one-year period, exponentially grew within the later 3 months, on the lower back region with surface ulceration. (See figure 1). The rest of his examination was unremarkable with all vital signs and labs were within normal limits. Past surgical history is comprised of a cholecystectomy, unknown date. Social history is comprised of a 55 smoking-year, with 5 years cessation since time of examination; no social history of drug or alcohol use in 25 years. Family history was noncontributory. There are no known drug allergies.

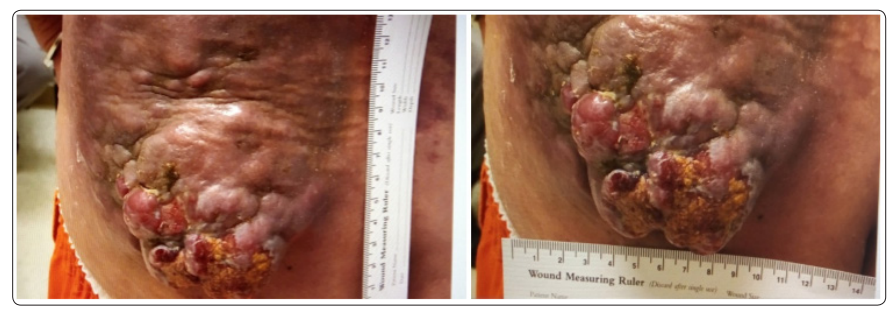

Figure 1: Gross presentation of violaceous \& necrotic ulcerative tumor

Patient was admitted to the hospital and underwent imaging and surgical resections. Pelvic MRI with and without contrast, showed a multilobulated enhancing soft tissue mass measuring 8.7 x 10.4 x 15.1 $\mathrm{cm}^{3}$ at the left gluteal region with exophytic extension through the dermis and directly abutting the left paraspinal muscles. Radiology differential diagnosis consisted of undifferentiated pleomorphic sarcoma and well-differentiated liposarcoma, given the dermal thickening overlying the mass. Previous biopsy showed benign features, thus, repeat biopsy was recommended. (See Figure 2). Sentinel lymph node biopsy and PET scan were not completed.
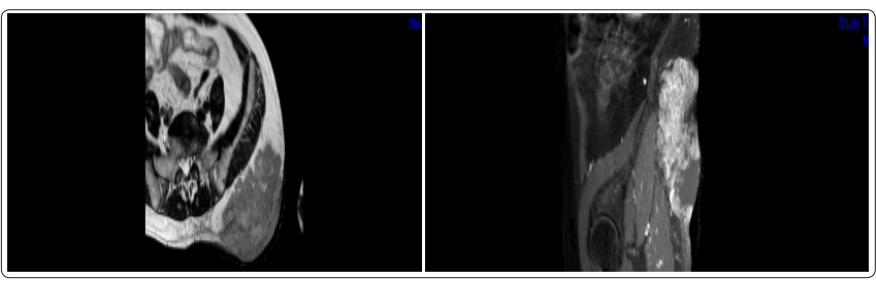

Figure 2: MRI with \& without contrast
Abdominal-pelvic CT with IV contrast was also completed, showing mild bilateral adrenal hypertrophy and nodularity, with no evidence of metastasis or lymphadenopathy. CT imaging was consistent with MRI reading of large left flank mass invading into the left gluteal and erector spinae muscles. (See Figure 3).
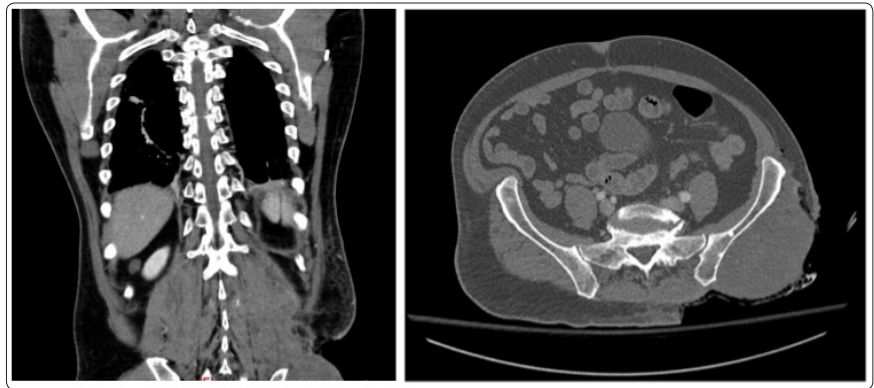

Figure 3: CT abdomen-pelvis with IV contrast

The patient was admitted, tangential surgical excision and debridement of left flank wound was performed with partial primary closure of the lateral and superior edges of the wound with drain placement and 1:1 meshed autograft approximately $400 \mathrm{~cm}^{2}$ placement under negative wound pressure device. (See Figure 4).

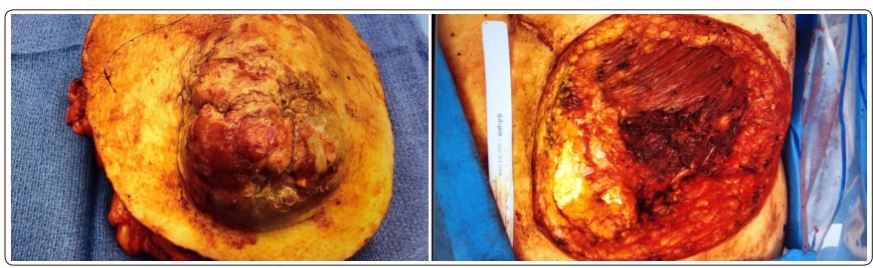

Figure 4: Post-surgical wide tangential excision $\&$ tumor resection

Specimen showed a soft tissue mass showing features of Merkel cell where tumor cells fills and expands the dermis with invasion of the subcutaneous with no perineural or vascular invasion seen (See figure 5). Immunoperoxidase stains show that the tumor cells show diffuse dot-like positivity with CK20 and are negative for CK7, there is diffuse positivity with NSE, synaptophysin and CD56 with strong diffuse Ki-67 positivity noted in $>65 \%$ of tumor cells (See figure 6). CD99 shows diffuse small faint dot-like paranuclear positivity. Tumor cells are negative for chromogranin A, EMA, TTF-1, CD117a, CD45, CD3, CD20, CD34, CD10, P63, HMB-45, and Melan A. (See figure 7). Patient was a lost to follow-up status post-resection.

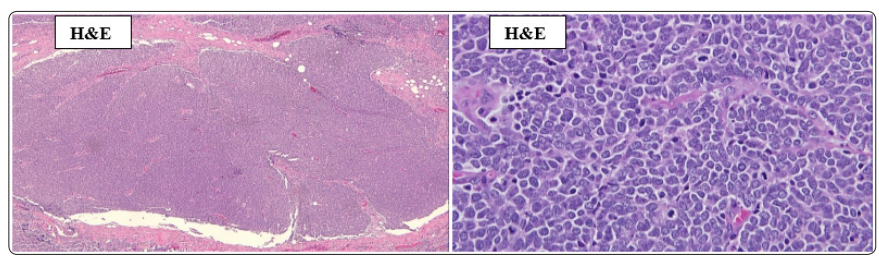

Figure 5: H\&E showing sheets of small 


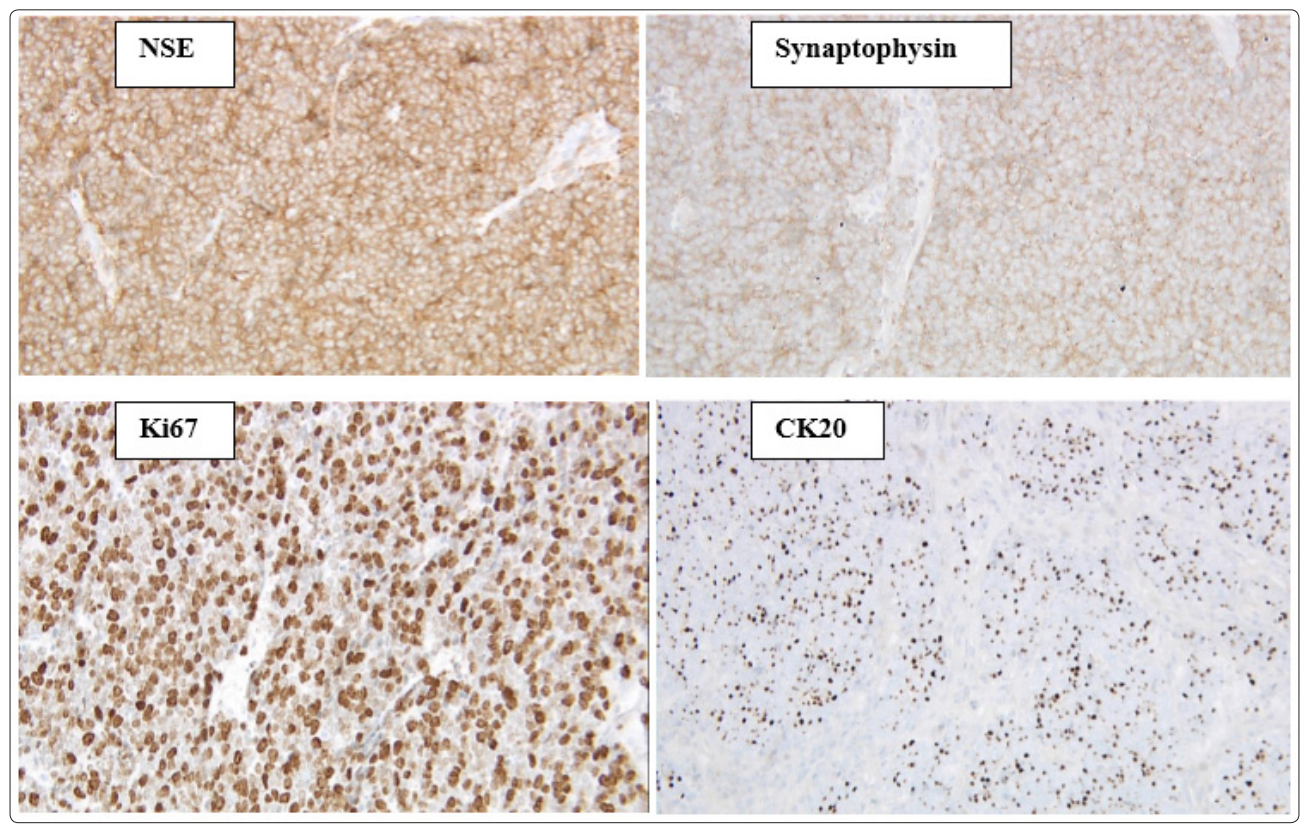

Figure 6: NSE, Synaptophysin, Ki67, \& CK20

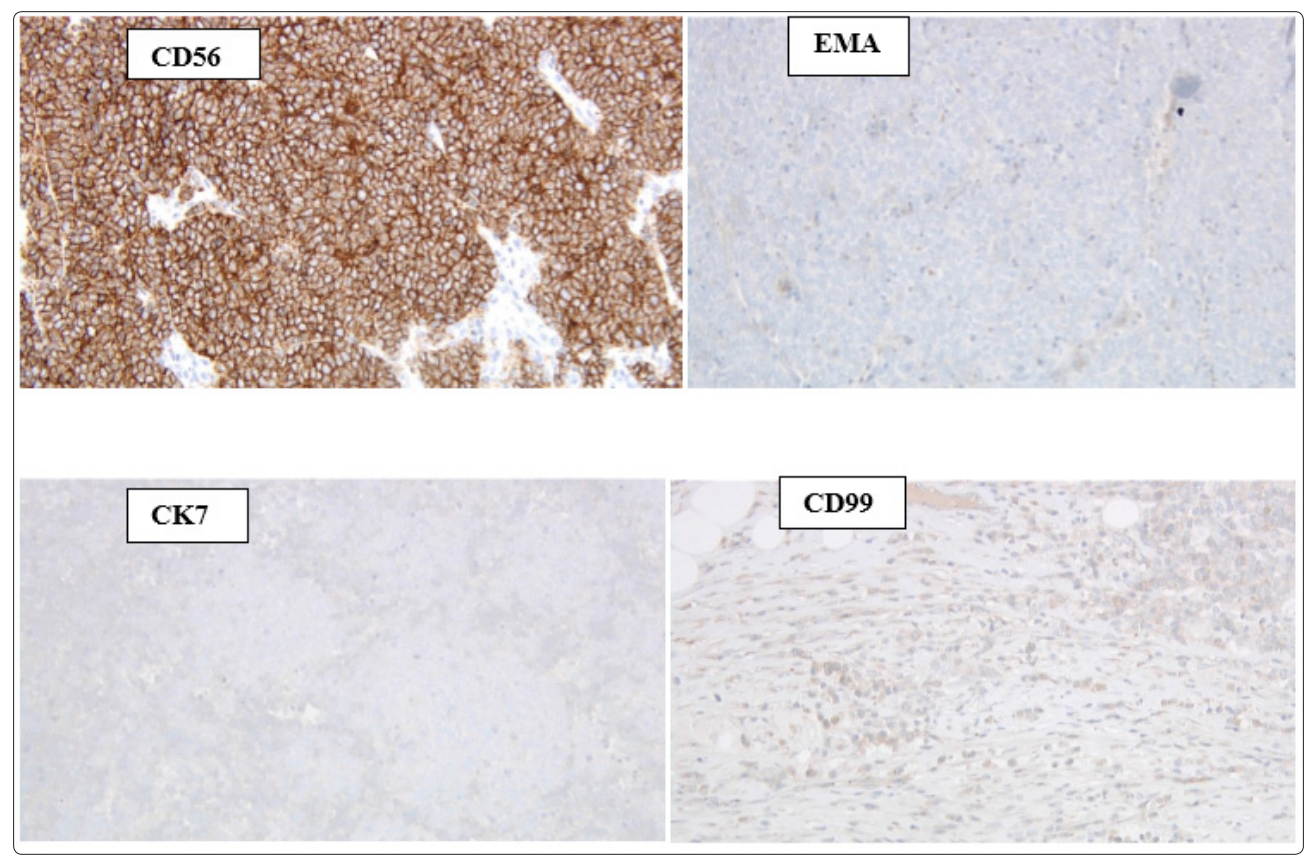

Figure 7: CD56, EMA, CK7, \& CD99

\section{Discussion}

Merkel cell carcinoma is a rare and indolent cancer, most often occurring in immuno-compromised patients (HIV/Hepatitis infections, organ transplant recipients or patients with Chronic lymphocytic leukemia) [6]. MCC is commonly associated with prolonged exposure to UV radiation, causing high mutational load within the genome. However, approximately $80 \%$ of the MCC cases are clonally associated with polyomavirus [7]. The patient discussed in this case report was tested for polyomavirus, and the status was negative. In addition, sun-exposure or prolonged UV radiation was not seen in the history, with no source of immunocompromisability. In the event of ruling out other etiologies, this concludes a molecular pathogenesis in the development of this virulent tumor in this patient [1].
Studies have shown that molecular tumorigenesis expression of genes, such as NOTCH1, NOTCH2, and JAG1, are associated with MCC polyomavirus negative patients, with JAG1 having a higher association and NOTCH having a better overall median survival [7]. Epigenetic changes associated with MCC, with decreased expression of $\mathrm{H} 3 \mathrm{~K} 27 \mathrm{~m}^{3}$, a histone modifier, has been shown in some studies, with higher association with MCC polyomavirus negative status, with squamous cell carcinoma (SCC) [8]. These molecular mutations can be seen, by morphologically differentiating the cellular structure and immunohistochemical (IHC) stain. One study showed a morphology of MCC polyomavirus negative pathologically similar to a large cell neuroendocrine carcinoma, with large prominent nucleoli, and abundant cytoplasm, with molecular expression of 
cytokeratin 7 and thyroid transcription factor 1, with absence or overexpression of $\mathrm{p} 53$. Contrarily, MCC polyomavirus positive expressed cytokeratin 8, 18, 20, CD99, and EMA were commonly expressed in MCC polyomavirus positive patients [9].

\section{Conclusion}

In such, molecular pathogenesis and tumorigenesis consideration is imperative in establishing treatment and management of patients with MCC polyomavirus negative status, of non-indolent proliferative type. Morphological pathology, as well as, IHC staining can further convey tumor virulence. More studies are required to risk stratify patients based on molecular pathogenesis and pathology in concluding overall survival and prognosis in patients with MCC polyomavirus negative status.

\section{References}

1. Coggshall K, Tello TL, North JP, Yu SS (2018) Merkel Cell Carcinoma: An update and review. Journal of the American Academy of Dermatology 78: 433-442.

2. Andea AA, Coit DG, Amin B, Busam KJ (2008) Merkel Cell Carcinoma. Cancer 113: 2549-2559.

3. National Comprehensive Cancer Network (2019) Merkel Cell Carcinoma, https://www.nccn.org/professionals/physician_gls/ pdf/mcc.pdf.
4. Pulitzer M (2017) Merkel Cell Carcinoma. Surgical Pathology Clinics 10: 399-408.

5. Howell RS, Rice JA, Sticco K, Donovan V, Castellano M, et al. (2018) An unusual presentation of Merkel Cell Carcinoma: a case-report. Journal of Surgical Case Reports 8: 1-3.

6. Hughes MP, Hardee ME, Cornelius LA, Hutchins LF, Becker JC, et al. (2014) Merkel Cell Carcinoma: Epidemiology, Target, and Therapy. Current Dermatology Reports 3: 46-53.

7. Wardhani LO, Matsushita M, Kuwamoto S, Nonaka D, Nagata $\mathrm{K}$, et al (2019) Expression of NOTCH 3 and Jagged 1 is associated with Merkel Cell Polyomavirus status and prognosis in Merkel Cell Carcinoma. Anticancer Research 39: 319-329.

8. Matsushita M, Iwasaki T, Wardhani LO, Kuwamoto S, Nonaka D, et al (2019) Decreased H3k27me3 expression is associated with Merkel Cell polyomavirus negative, Merkel Cell Carcinoma, especially combined cutaneous Squamous Cell Carcinoma. Anticancer Research 39: 5573-5579.

9. Karverrac T, Tallet A, Miquelestorena-Standley E, Houben R, Schrama D, et al. (2019) Morphologic and immunophenotypical features distinguishing Merkel Cell polyomavirus-positive and negative Merkel Cell Carcinoma. Modern Pathology 32: $1605-$ 1616.
Copyright: (02020 West Michele. This is an open-access article distributed under the terms of the Creative Commons Attribution License, which permits unrestricted use, distribution, and reproduction in any medium, provided the original author and source are credited. 